\title{
Added lower limb mass does not affect biomechanical asymmetry but increases metabolic power in runners with a unilateral transtibial amputation
}

\author{
Ryan S. Alcantara • Owen N. Beck • Alena M. Grabowski
}

Twitter: RA - @Ryan_Alcantara_, OB - @Owen1Beck, AG - @AlenaGrabowski

\begin{abstract}
Purpose:We determined the metabolic and 2 biomechanical effects of adding mass to the runningspecific prosthesis (RSP) and biological foot of indi4 viduals with a unilateral transtibial amputation (TTA) during running. Methods: 10 individuals (8 males, 2 females) with a TTA ran on a force-measuring treadmill at $2.5 \mathrm{~m} / \mathrm{s}$ with $100 \mathrm{~g}$ and $300 \mathrm{~g}$ added to their

8 RSP alone or to their RSP and biological foot while we measured their metabolic rates and calculated peak

10 vGRF, stance-average vGRF, and step time symmetry indices. Results: For every $100 \mathrm{~g}$ added to the RSP 12 alone, metabolic power increased by $0.86 \%(\mathrm{p}=0.007)$ and for every $100 \mathrm{~g}$ added to the RSP and biological
\end{abstract}

Corresponding Author: Ryan S. Alcantara

Department of Integrative Physiology

University of Colorad Boulder

$354 \mathrm{UCB}$

Boulder, CO, 80309-0354, USA

Tel.: +1-303-492-7333

E-mail: ryan.alcantara@colorado.edu

Owen N. Beck

The George W. Woodruff School of Mechanical Engineering School of Biological Sciences

Georgia Institute of Technology

Atlanta, GA, USA

Alenâ M. Grabowski

Department of Integrative Physiology

University of Colorado Boulder

Boulder, CO, USA

Department of Veterans Affairs

Eastern Colorado Healthcare System

Denver, CO, USA

All authors consent to the sharing of this preprint on SportRxiv - Cite as: Alcantara, RS, Beck ON, Grabowski AM (2019). Added lower limb mass does not affect biomechanical asymmetry but increases metabolic power in runners with a unilateral transtibial amputation. SportRxiv. https://doi.org/10.31236/osf.io/xcus7

foot, metabolic power increased by $1.74 \%(\mathrm{p}<0.001)$ during running. Adding mass had no effect on peak vGRF ( $\mathrm{p}=0.102)$, stance-average vGRF $(\mathrm{p}=0.675)$, or step time $(p=0.413)$ symmetry indices. We also found that the swing time of the affected leg was significantly shorter than the unaffected leg across conditions $(\mathrm{p}<0.007)$. Conclusions: Adding mass to the lower limbs of runners with a TTA increased metabolic power by more than $1 \%$, which has been reported for those without an amputation. We found no effect of added mass on biomechanical asymmetry, but the affected leg had consistently shorter swing times than the unaffected leg. This suggests that individuals with a TTA maintain biomechanical asymmetries despite changes in RSP mass and that lightweight prostheses could improve performance by minimizing metabolic power without affecting biomechanical asymmetry.

Keywords amputee · symmetry · energetics · economy $\cdot$ prosthesis

\section{Introduction}

Metabolic power and oxygen consumption increase $1 \%$ for every $100 \mathrm{~g}$ added to each foot/ankle of runners without amputations across speeds ranging from 3.35$4.88 \mathrm{~m} / \mathrm{s}[1-9]$ and increased metabolic power decreases $3000 \mathrm{~m}$ running performance [3]. While the directional changes between metabolic power and distance running performance are likely the same for individuals with and without a transtibial amputation (TTA), the effects of added lower limb mass on metabolic power for individuals with a TTA is unknown.

Most individuals with a TTA run using a runningspecific prosthesis (RSP), which is a passive-elastic carbon fiber device that lacks an ankle joint and is at- 
tached to a carbon fiber socket that surrounds the resid-

48 ual limb. The metabolic effects of increasing lower limb mass have been investigated in individuals without a

50 TTA [10-14], but the use of an RSP in individuals with a TTA likely influences the effect of lower limb mass on 52 metabolic power. Because an RSP has approximately half the mass of a biological foot and shank $[15,16]$, 54 adding mass to the RSP and biological foot of an individual with a TTA would result in a relatively larger

56 increase in the mass of their affected leg (AL) compared to their unaffected leg (UL). Thus, adding mass to the

58 RSP may increase metabolic power by a greater amount in individuals with a TTA compared to values reported ${ }_{60}$ in individuals without a TTA.

The structural and functional differences between 62 the biological foot and ankle and RSP of an individual with a TTA result in asymmetric running biomechanics individuals with unilateral TTAs used a recommended average vertical ground reaction forces and longer grou

6 contact times compared to their UL across a wide range of running speeds (3m/s - top speed) [17, 18,21]. In in-

70 dividuals with and without a TTA, asymmetric running biomechanics have been identified as risk factors for in-

72 jury [22,23] and increase metabolic cost [24]. However, adding mass to the RSP of individuals with a TTA may 74 not affect the biomechanics of the AL. For example, Grabowski et al. (2009) found that adding 100 and 300

${ }^{6} \mathrm{~g}$ to the RSP of sprinters with a TTA had no effect on leg swing time, stance-average vGRF, or top speed [21].

When a prosthetist prescribes an RSP to an individual with a TTA, they typically adjust prosthe-

so sis height, stiffness, and alignment to reduce kinematic asymmetries such as step frequency between legs [25].

82 However, prosthetists are not often equipped to detect and minimize asymmetries in variables such as

84 peak or stance-average vertical ground reaction force (vGRF), which have been associated with reductions

${ }_{86}$ in metabolic cost during running [24]. Decreases in peak and stance-average vGRF asymmetry have been achieved

8 by changing RSP model and height [24], but adding relatively small $(\leq 300 \mathrm{~g})$ amounts of mass to the RSP

90 could increase the $\mathrm{AL}$ peak and stance-average vGRF [26], decrease asymmetry, and potentially lower metabolic

92 power during running. Determining how RSP mass affects metabolic cost during running may therefore in-

94 form RSP design and rehabilitation strategies seeking to reduce running injury prevalence and improve run96 ning performance in individuals with a TTA.

We investigated the metabolic and biomechanical 98 effects of adding mass to the RSP alone or to the RSP and biological foot of individuals with a TTA during running. We hypothesized that adding mass to the RSP alone would decrease peak vGRF, stance-average vGRF, and step time asymmetry. Decreased biomechanical asym- 102 metry in individuals with a TTA could potentially improve metabolic cost [24], but adding mass to the RSP alone may increase metabolic power due to the additional metabolic energy required to support the increased body weight and to swing a heavier leg. Thus, we hypothesized that adding mass to the RSP alone would have no effect on metabolic power due the offsetting effects of symmetry and biomechanical changes that increase metabolic power when running with added mass. We also hypothesized that mass added to the RSP and biological foot would have no effect on peak vGRF, stance-average vGRF, or step time asymmetry, and would increase metabolic power during running.

\section{Methods}

2.1 Participants

Ten individuals ( 8 males, 2 females; mean \pm SD: mass $70.3 \pm 8.3 \mathrm{~kg}$, height $1.76 \pm 0.09 \mathrm{~m}$, age $38 \pm 5$ years $)$ with a TTA participated. All participants had at least one year of experience using an RSP and reported running at least 3 days per week over the 6 months prior to data collection. The protocol was approved by the University of Colorado Boulder Institutional Review Board and all participants provided informed consent prior to participation.

\subsection{Experimental Protocol}

Each participant ran on a force-measuring treadmill (1000 Hz; Treadmetrix, Park City, UT) while we measured their rates of oxygen consumption and carbon dioxide production via indirect calorimetry (ParvoMedics TrueOne 2400, Sandy, UT). Participants were instructed to refrain from eating food or drinking anything but water for the 2 hours leading up to data collection. Following a 5-minute warm-up on the treadmill, participants ran at $2.5 \mathrm{~m} / \mathrm{s}$ for 5 minutes under 5 conditions in a random order: no added mass, $100 \mathrm{~g}$ added to the RSP alone, $300 \mathrm{~g}$ added to the RSP alone, $100 \mathrm{~g}$ added to the RSP and biological foot (200 g total), and 300 $\mathrm{g}$ added to the RSP and biological foot (600 g total). Participants were given at least 5 minutes rest between conditions. During each trial we monitored respiratory exchange ratios (RERs) and ensured that participants maintained primarily aerobic metabolism, indicated by an RER < 1.0. We added $100 \mathrm{~g}$ and $300 \mathrm{~g}$ to allow for comparison with prior work $[2,3,21]$. We adhered the 


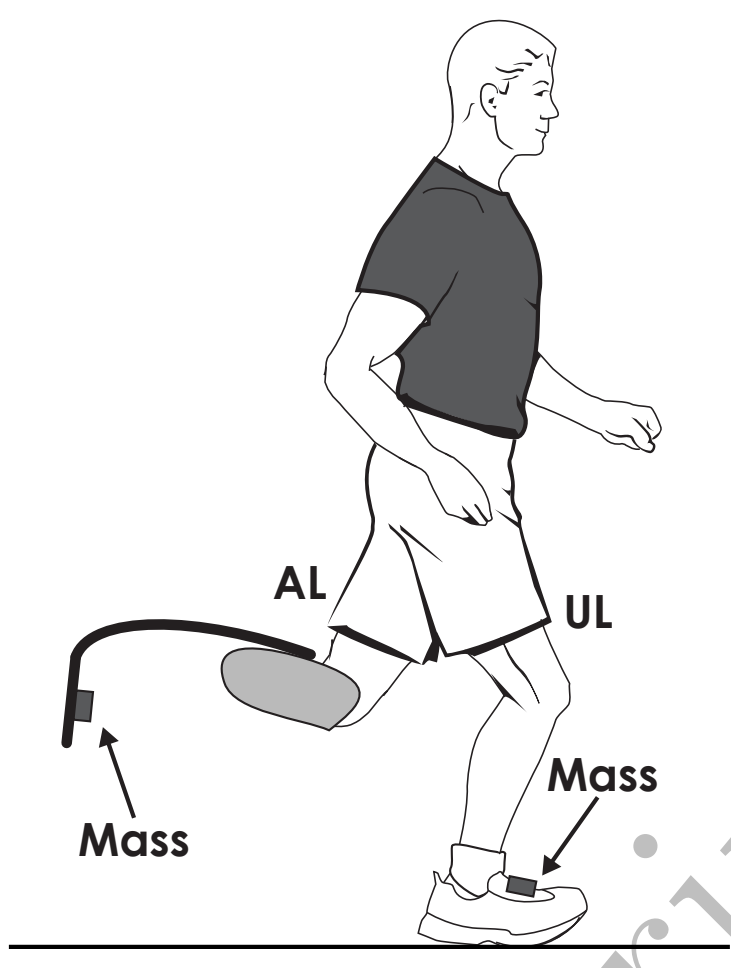

Fig. 1 The center of the added mass was adhered $10 \mathrm{~cm}$ from the distal end of the shoe on the unaffected leg (UL) and of the RSP on the affected leg (AL).

center of $100 \mathrm{~g}(10 \mathrm{~cm} \mathrm{x} 5 \mathrm{~cm} \mathrm{x} 2 \mathrm{~cm})$ and $300 \mathrm{~g}(10$ $\mathrm{cm} \times 10 \mathrm{~cm} \times 3 \mathrm{~cm}) 10 \mathrm{~cm}$ from the distal end of the RSP or shoe (Fig. 1).

\subsection{Analysis}

We averaged rates of oxygen consumption and carbon dioxide production over the last two minutes of each trial, calculated gross metabolic power [27], and nor-

154 malized metabolic power to each participant's body mass including their RSP and running clothes, but ex-

156 cluding the $100 \mathrm{~g}$ or $300 \mathrm{~g}$ added mass. At most, the added mass constituted $\sim 1 \%$ of a runner's body mass, and prior studies have not included this in the normalization of metabolic power $[2,3,9]$. We measured ground reaction forces for 30 seconds during the final minute of each condition. We used a custom Matlab script (Mathworks, Natick, MA, USA) to filter GRF data using a zero-lag 4th-order low-pass Butterworth

164 filter with a $30 \mathrm{~Hz}$ cut-off and used ten steps from each leg for analyses. We defined stance phase as the period when the runner's vGRF exceeded a $20 \mathrm{~N}$ threshold. Stance-average vGRF was normalized to body weight and calculated as the mean vGRF during ground contact for each of the respective leg's ten steps. We used the absolute value of the symmetry index (SI) to determine peak vGRF, stance-average vGRF, and step time asymmetry between the AL and UL [28]. Symmetry Index is represented as a percentage (Eq. 1) where perfect symmetry between legs is $0 \%$ :

$S I=\left|\frac{U L-A L}{0.5(U L+A L)}\right| * 100$

We constructed linear mixed-effects models $(\alpha=$ $0.05)$ to determine the effect of added mass on gross metabolic power, peak vGRF SI, stance-average yGRF SI, and step time SI during running. Non-statistically significant model coefficients were removed from the model on the basis that the coefficient was not significantly different than 0 . Unstandardized model coefficients are reported in captions of Figures 2 and 3. We analyzed data in $\mathrm{R}$ (version 3.5.1) [29] using custom scripts and packages $[30,31]$.

\section{Results}

The mean ( $\pm \mathrm{SE}$ ) metabolic power of running at 2.5 $\mathrm{m} / \mathrm{s}$ with no added mass was $11.97 \pm 0.35 \mathrm{~W} / \mathrm{kg}$. For every $100 \mathrm{~g}$ added to the RSP alone, gross metabolic power increased $0.86 \pm 0.25 \%(\mathrm{p}=0.007$; Fig. 2$)$. When mass was added to the RSP and biological foot, the effect approximately doubled as gross metabolic power increased by $1.74 \pm 0.25 \%$ per $100 \mathrm{~g}$ added to each limb $(\mathrm{p}<0.001$; Fig. 2).

When running with no added mass, participants had a mean $( \pm \mathrm{SE})$ stance-average vGRF SI of $7.39 \pm$ $2.03 \%$, peak vGRF SI of $13.95 \pm 2.63 \%$, and step time SI of $5.77 \pm 1.58 \%$. There was no significant change in symmetry indices regardless of the amount of mass added (100 or $300 \mathrm{~g}$ ) or limb(s) mass was added to (RSP alone or RSP and biological foot). There were no differences in stance-average vGRF SI, peak vGRF SI, or step time SI between mass added to the RSP alone or mass added to the RSP and biological foot $(\mathrm{p}=0.389$, $\mathrm{p}=0.442$, and $\mathrm{p}=0.579$, respectively). Further, there were no effects of added mass on stance-average vGRF SI $(p=0.675)$, peak vGRF SI $(p=0.102)$, or step time SI $(\mathrm{p}=0.413$; Table 1$)$.

We also performed a post hoc analysis to determine if adding mass to the lower limb affects leg swing time in individuals with a TTA. When running with no additional mass, participants had a mean $( \pm$ SE) leg swing time of $459 \pm 16 \mathrm{~ms}$ for the $\mathrm{AL}$ and $468 \pm 18 \mathrm{~ms}$ for the UL. This difference between leg swing times was statistically significant and persisted across added mass 
Table 1 Mean ( \pm SE) symmetry Index (SI) for stance-average vertical ground reaction force (vGRF), peak vGRF, and step time across all conditions. There were no significant differences ( $p>0.05)$ between adding mass to the running specific prosthesis (RSP) alone or adding mass to the RSP and biological foot (both). There were no significant changes in stance-average vGRF, peak vGRF, or step time SI across added mass conditions ( $\mathrm{p}>0.05)$.

\begin{tabular}{llccc} 
& & \multicolumn{3}{c}{ Mass Added per Limb } \\
Symmetry Index & Location & $0 \mathrm{~g}$ & $100 \mathrm{~g}$ & $300 \mathrm{~g}$ \\
\hline \multirow{2}{*}{ Stance-Average vGRF } & RSP & \multirow{2}{*}{$7.39 \pm 2.03 \%$} & $8.35 \pm 1.95 \%$ & $7.59 \pm 2.06 \%$ \\
& Both & & $7.54 \pm 2.16 \%$ & $7.68 \pm 2.01 \%$ \\
\hline \multirow{2}{*}{ Peak vGRF } & RSP & $13.95 \pm 2.63 \%$ & $14.01 \pm 3.03 \%$ & $12.33 \pm 2.82 \%$ \\
& Both & & $13.40 \pm 2.79 \%$ & $13.91 \pm 2.43 \%$ \\
\hline \multirow{2}{*}{ Step Time } & RSP & $5.77 \pm 1.58 \%$ & $5.75 \pm 1.36 \%$ & $5.23 \pm 1.29 \%$ \\
& Both & & $5.66 \pm 1.55 \%$ & $5.77 \pm 1.48 \%$ \\
\hline
\end{tabular}

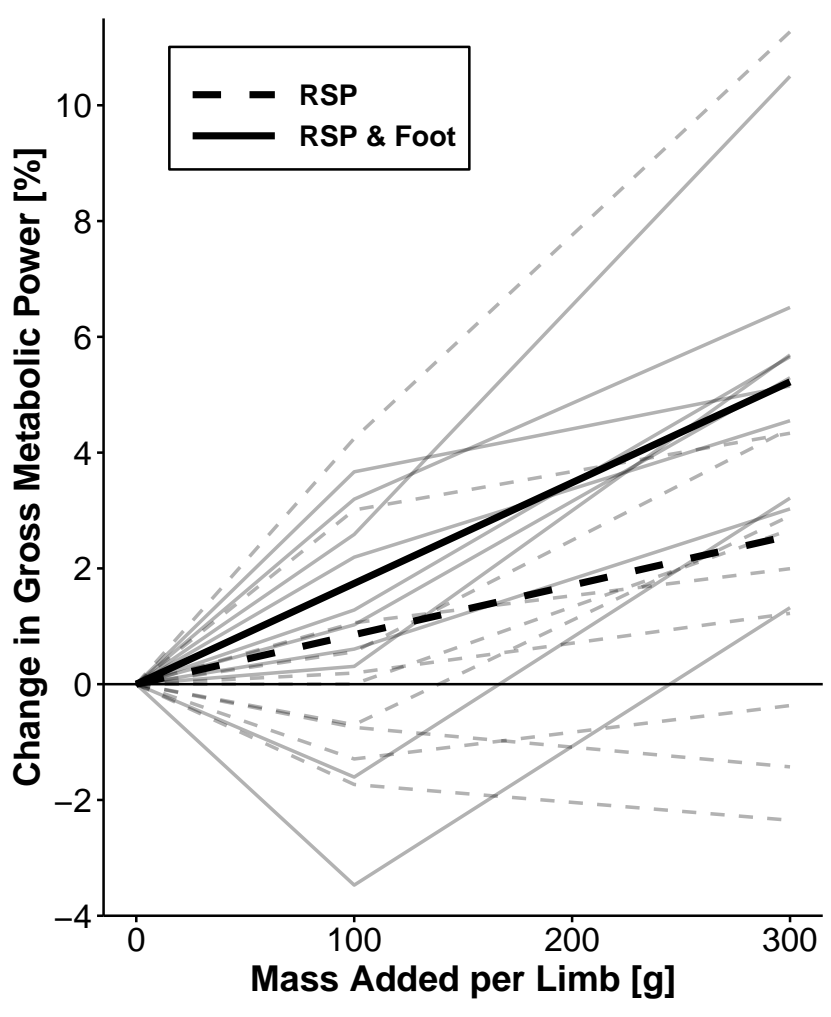

Fig. 2 Percentage change in gross metabolic power for running at $2.5 \mathrm{~m} / \mathrm{s}$ without added mass and with added mass of 100 and $300 \mathrm{~g}$. Mass added to the RSP alone resulted in a $0.86 \%$ increase in metabolic power $(\mathrm{W} / \mathrm{kg})$ per $100 \mathrm{~g}$ $\left(\mathrm{p}=0.007\right.$; Metabolic Power $[\mathrm{W} / \mathrm{kg}]=11.947+0.001^{*}$ mass [g]). Mass added to the biological foot and RSP resulted in a $1.74 \%$ increase in metabolic power per $100 \mathrm{~g}$ added to each foot $(\mathrm{p}<0.001 ;$ Metabolic Power $[\mathrm{W} / \mathrm{kg}]=11.924+$ $0.002 *$ mass $[\mathrm{g}])$. Thin lines represent subject-specific data, where the dashed lines indicate mass added to the RSP alone and the solid lines indicate mass added to RSP and biological foot. Thick lines represent model overall predictions across conditions. conditions. When we added mass to the RSP and biological foot, UL swing time was $10 \pm 3$ ms greater than AL swing time $(\mathrm{p}=0.007)$, but there was no effect of added mass on leg swing time $(\mathrm{p}=0.228)$. When we added mass to the RSP alone, UL swing time was 10 $\pm 3 \mathrm{~ms}$ greater than AL swing time $(\mathrm{p}=0.005)$ and for every $100 \mathrm{~g}$ added to the RSP alone, swing time for both legs increased by $5 \pm 1 \mathrm{~ms}(\mathrm{p}=0.001$; Fig. 3$)$.

\section{Discussion}

We reject our first and second hypotheses that mass added to the RSP alone would decrease peak vGRF asymmetry, stance-average vGRF, and step time asymmetry and have no effect on metabolic power during running. Although adding mass to the RSP alone reduced the mass discrepancy between the AL and UL, adding 100-300 $\mathrm{g}$ to the RSP had no effect on biomechanical asymmetry but increased metabolic power by $0.86 \%$ per 100 g added. While adding mass to the RSP alone did not reduce metabolic power, Beck et al. (2017) found that an optimal combination of RSP model, stiffness, and height increased peak vGRF symmetry and decreased metabolic cost of transport during running at $2.5-3.0 \mathrm{~m} / \mathrm{s}[24]$. Our data suggest that RSP mass does not affect these measures of symmetry, as participants maintained asymmetric peak vGRF, stanceaverage vGRF, and leg swing time despite running with up to $300 \mathrm{~g}$ added to their RSP. While RSP mass had no effect on biomechanical asymmetry, we found that increasing RSP mass increased metabolic power during running.

Adding mass to the RSP and biological foot had no effect on peak vGRF, stance-average vGRF, or step time asymmetry, but increased metabolic power by $1.74 \%$ per $100 \mathrm{~g}$ added to each limb (RSP and biological foot), which is greater than the reported $\sim 1 \%$ increase in metabolic power per $100 \mathrm{~g}$ added to each foot or ankle 


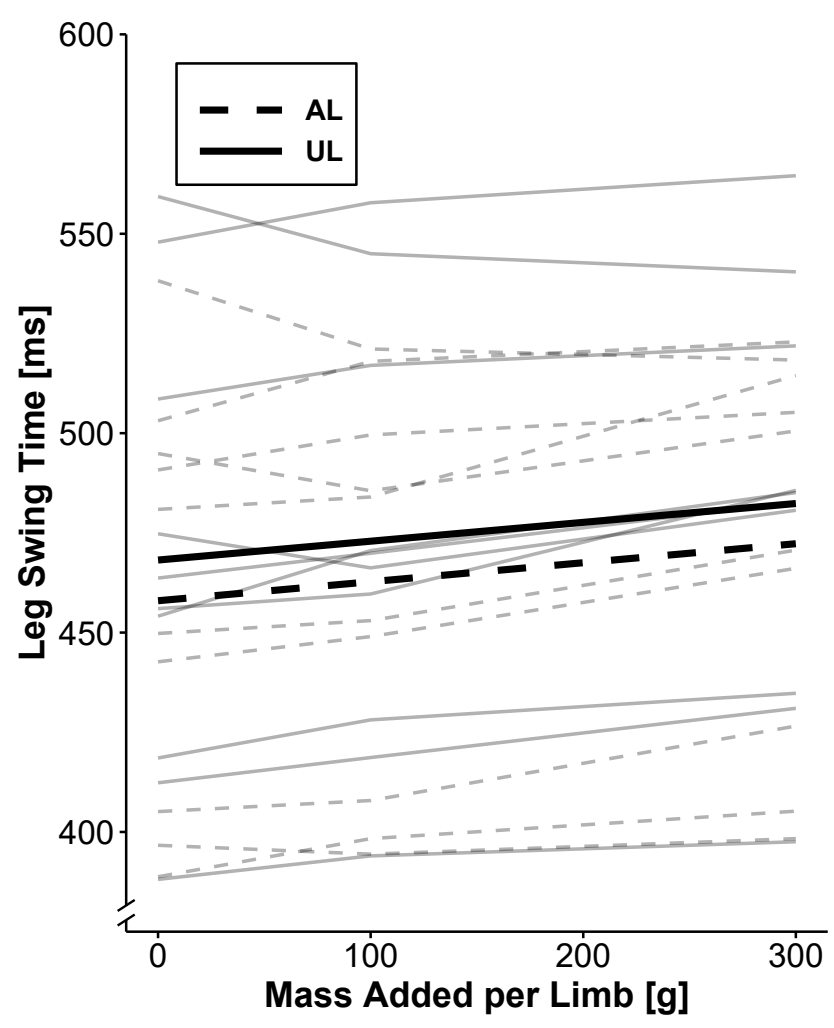

Fig. 3 Swing time of the affected leg (AL) and unaffected leg (UL) for running at $2.5 \mathrm{~m} / \mathrm{s}$ without added mass and with added mass of 100 and $300 \mathrm{~g}$ to the RSP alone. Across the added mass conditions, UL swing time was greater than AL swing time $(\mathrm{p}=0.005)$, and swing time of both legs increased by $5 \mathrm{~ms}$ per $100 \mathrm{~g}(\mathrm{p}=0.001$; Swing time $[\mathrm{ms}]=458.01+$ $0.0475 *$ mass $[\mathrm{g}]+10.148 *$ leg [AL: 0, UL: 1]). Thin lines represent subject-specific data, where the dashed lines indicate the AL and the solid lines indicate the UL. Thick lines represent model overall predictions across conditions.

in runners without a TTA $[1-3,5-9]$. Our findings supour third and fourth hypotheses that mass added to the RSP and biological foot would have no effect on biomechanical asymmetry and that runners with a TTA would experience an increase in metabolic power when mass is added to the RSP and biological foot. Further, our data suggest that individuals with a transtibial amputation may be more metabolically sensitive to mass added to their RSP and biological foot compared to individuals without an amputation.

AL and UL swing time did not change with mass added to the RSP and biological foot, but UL swing time was consistently greater than AL swing time re264 gardless of whether mass was added or not. The swing time of both legs increased as mass was added to the

${ }_{266}$ RSP alone. Thus, adding mass to the RSP alone increases AL swing time but participants increased UL swing time and thereby maintained leg swing time asymmetry. Grabowski et al. (2009) found that swing time between the AL and UL did not differ when adding 100 and $300 \mathrm{~g}$ to the RSP compared to no added mass at running speeds of $3 \mathrm{~m} / \mathrm{s}$ up to maximum speed [21]. However, we found that UL swing times were consistently greater than AL swing times across all conditions at $2.5 \mathrm{~m} / \mathrm{s}(0,100$, and $300 \mathrm{~g}$ added to RSP $)$. It is possible that different results between studies are due to differences in statistical power; as Grabowski et al. (2009) state that they may have had limited statistical power due to including six participants in their study [21]. Our data suggest that RSP mass has an effect on leg swing time and that swing times differ between the legs of individuals with a TTA without added mass and when up to $300 \mathrm{~g}$ is added to the RSP.

The location of mass added to a runner's body has a differential effect on metabolic cost during running. Teunissen et al. (2007) found that when adding weight, the product of mass and gravitational acceleration, around the waist, every $10 \%$ increase in body mass ( $\sim 6 \mathrm{~kg})$ increased net metabolic power (calculated by subtracting the metabolic power required to stand from the metabolic power measured during a given condition) by $\sim 13 \%$ to run at $3.0 \mathrm{~m} / \mathrm{s}$ [32]. The greater metabolic cost to run was primarily attributed to the increased force generation required to support body weight $[33,34]$. We extrapolated these findings to determine the effect of adding $600 \mathrm{~g}$ to the waist, which is $\sim 1 \%$ of the average participant body mass, and found that every $1 \%$ increase in body weight around the waist would increase net metabolic power by $\sim 1.3 \%$. However, if a similar mass is added to the lower limbs (300 $\mathrm{g}$ on each foot) of runners without a TTA, prior work suggests that metabolic power would increase by $\sim 3 \%$ and our findings suggest that metabolic power would increase by $5.2 \%$ in runners with a TTA. Further, the mass location affects the time during a stride that the metabolic penalty occurs. For example, when mass is added to the waist, the additional metabolic cost occurs during stance phase due to the additional force that the legs must produce to support the weight of the body during running [32-35]. But when mass is added to the feet, the legs need support the increased body weight and raise the foot with the added mass off the ground, swing it forward, and decelerate it before contacting the ground again $[1,9,13]$. Thus, added mass to the feet incurs an additional metabolic cost during stance and swing phase.

Adding mass to the feet effects the lower leg's moment of inertia, which influences the metabolic energy required to swing the leg during running $[6,9,13]$. We found that runners with a TTA have a larger increase in metabolic power due to mass added to their RSP and biological foot compared to runners without a TTA 
(1.74\% vs. $1 \%$ increase per $100 \mathrm{~g}$ on each foot [1-4]. 324 Individuals with a TTA have approximately half the mass below the knee on their AL compared to a biorepresents a larger relative increase in the lower leg's requires more metabolic energy to swing the leg. Using previously published inertial properties for an RSP, socket, and residual limb of a sprinter with TTAs [15], RSP would increase the AL's moment of inertia by ap334 proximately $9 \%$ compared to $4 \%$ in an individual without a TTA. While the effects of leg moment of inertia on metabolic cost and muscle activation have been examined during walking in individuals with and without a TTA $[10,36,37]$, to our knowledge no studies have quantified the relationship between lower leg moment of inertia and metabolic cost during running in individuals with a TTA. We suspect that the greater metabolic 342 costs incurred by individuals with a TTA when mass is added to the RSP are due to these disproportionately

344 larger inertial loads, but this requires further investigation.

We found that adding mass to the RSP alone or to the RSP and biological foot of rumners with a TTA

348 had no effect on peak vGRF, stance-average vGRF, and step time asymmetry. It is possible that the mass added to the RSP in the present study was simply not enough to elicit changes in kinetic or kinematic asymmetry.

352 However, we do not suspect that adding larger amounts of mass to the RSP would decrease biomechanical asymmetries enough to offset the increased metabolic power associated with the added mass. Prior work found that 356 a $10 \%$ decrease in peak vGRF SI correlated to a $1.9 \%$ decrease in net metabolic cost of transport (energy ex-

358 penditure per unit distance instead of unit time) in runners with a TTA [24], but when we added $300 \mathrm{~g}$ to the RSP, there was no effect on peak vGRF SI and gross metabolic power increased by $\sim 2.6 \%$. Thus, it is likely by the metabolic cost of running with an additional mass on the RSP.

We chose to add $100 \mathrm{~g}$ and $300 \mathrm{~g}$ to the RSP and biological foot of our participants in order to make direct comparisons to prior work [1-3]. A future study comparing the metabolic effects of adding a proportional amount of mass to the legs of runners with and with-

370 out a TTA could further elucidate the effect of added mass on runner's feet by effectively normalizing to lower

372 leg mass. However, small amounts of mass may only be realistically added to the RSP before the socket fit is 374 compromised and becomes unattached from the residual limb during running. Previous work has determined the metabolic effect of adding mass to the shoes of individuals who ran at faster speeds $(3.35-4.88 \mathrm{~m} / \mathrm{s})$ than the speed we tested $(2.5 \mathrm{~m} / \mathrm{s})$ [1-4], which may limit the generalizability of our findings. Frederick et al. (1984) found that the relative metabolic effect of added mass may decrease with faster speeds [1]. While other previous studies have found that metabolic cost increases $\sim 1 \%$ for every $100 \mathrm{~g}$ added to each foot at speeds from $3.35-3.61 \mathrm{~m} / \mathrm{s}[2-4]$, it is possible that runners with a TTA may experience a reduced effect of added mass at speeds faster than $2.5 \mathrm{~m} / \mathrm{s}$. Further studies are required to better understand the effect of added mass on metabolic power across a wide range of running speeds.

\section{Conclusions}

Adding 100 and $300 \mathrm{~g}$ to the RSP alone increased metaboliç90 power by $0.86 \%$ per $100 \mathrm{~g}$ and had no effect on stanceaverage vGRF, peak vGRF, or step time asymmetry. Adding 100 and $300 \mathrm{~g}$ to the RSP and biological foot of runners with a TTA increased metabolic power by $1.74 \%$ per $100 \mathrm{~g}$ on each leg. The swing time of the UL in runners with a TTA was greater than the swing time of the AL across all conditions and adding mass to the RSP alone increased the swing time of both legs proportionally. Adding mass to the RSP and biological foot had no effect on stance-average vGRF, peak vGRF, or step time asymmetry. Thus, adding mass to the RSP alone does not decrease asymmetry and would likely worsen distance running performance due to the associated increase in metabolic power. In contrast, reducing RSP mass may improve distance running performance by reducing metabolic power while having no effect on biomechanical asymmetry.

\section{Funding Statement}

This study was supportred by the University of Colorado Beverly Sears Graduate Student Grant.

\section{Conflict of interest}

The authors declare that they have no conflict of interest.

\section{References}

1. E. Frederick, J. Daniels, J. Hayes, The effect of shoe weight on the aerobic demands of running (na, 1984)

2. J.R. Franz, C.M. Wierzbinski, R. Kram, Medicine \& Science in Sports \& Exercise 44(8), 1519 (2012) 412 
3. W. Hoogkamer, S. Kipp, B.A. Spiering, R. Kram, Med Sci Sports Exerc 48(11), 2175 (2016)

4. C. Divert, G. Mornieux, P. Freychat, L. Baly, F. Mayer, A. Belli, International journal of sports medicine $\mathbf{2 9}(06)$, $512(2008)$

5. J.T. Fuller, C.R. Bellenger, D. Thewlis, M.D. Tsiros, J.D. Buckley, Sports medicine 45(3), 411 (2015)

6. P.E. Martin, Medicine and Science in Sports and Exercise $\mathbf{1 7}(4), 427$ (1985)

7. B.H. Jones, M.M. Toner, W.L. Daniels, J.J. Knapik, Ergonomics 27(8), 895 (1984)

8. A.D. Claremont, S.J. Hall, Medicine and Science in Sports and Exercise 20(2), 167 (1988)

9. M. Myers, K. Steudel, Journal of Experimental Biology 116(1), 363 (1985)

10. J.D. Smith, P.E. Martin, Journal of Applied Biomechanics 29(3), 317 (2013)

11. S.J. Mattes, P.E. Martin, T.D. Royer, Archives of physical medicine and rehabilitation 81(5), 561 (2000)

12. R.C. Browning, J.R. Modica, R. Kram, A. Goswami, Medicine \& Science in Sports \& Exercise 39(3), 515 (2007)

13. J.R. Modica, R. Kram, Journal of Applied Physiology 98(6), 2126 (2005)

14. B. Moed, R. Kram, in ISB XXth Congress-ASB 29th Annual Meeting, Cleveland, $O H$ (2005)

15. G.P. Brüggemann, A. Arampatzis, F. Emrich, W. Potthast, Sports Technology 1(4-5), 220 (2008)

16. P. De Leva, Journal of biomechanics 29(9), 1223 (1996)

17. O.N. Beck, A.M. Grabowski, Journal of Applied Physiology 124(3), 641 (2017)

18. B.S. Baum, H. Hobara, Y.H. Kim, J.K. Shim, Journal of applied biomechanics 32(3), 287 (2016)

19. C.J. Arellano, W.J. McDermott, R. Kram, A.M. Grabowski, PloS one 10(1), e0115637 (2015)

20. C.P. McGowan, A.M. Grabowski, W.J. McDermott, H.M. Herr, R. Kram, Journal of the Royal Society Interface $\mathbf{9}(73), 1975$ (2012)

21. A.M. Grabowski, C.P. McGowan, W.J. McDermott, M.T. Beale, R. Kram, H.M. Herr, Biology letters 6(2), $201(2009)$

22. C.H. Lloyd, S.J. Stanhope, I.S. Davis, T.D Royer, Gait \& posture 32(3), 296 (2010)

23. C. Daly, U. McCarthy Persson, R. Twycross-Lewis, R. Woledge, D. Morrissey, Scandinavian journal of medicine \& science in sports 26(4), 413 (2016)

24. O.N. Beck, P. Taboga, A.M.Grabowski, Journal of Applied Physiology 123(1), 38 (2017)

25. F. Innovations, Irvine, CA: Freedom Innovations (2014)

26. K.P. Clark, L.J. Ryan, P.G. Weyand, Journal of Experimental Biology 220(2), 247 (2017)

27. J. Brockway, Human nutrition. Clinical nutrition 41(6), 463 (1987)

28. W. Herzog, B.M. Nigg, L.J. Read, E. Olsson, Med Sci Sports Exerc 21(1), 110 (1989)

29. $\mathrm{R}$ Core Team, $R$ : A Language and Environment for Statistical Computing. R Foundation for Statistical Computing, Vienna, Austria (2019). URL https://www.Rproject.org/

30. J. Pinheiro, D. Bates, S. DebRoy, D. Sarkar. R core team (2018). nlme: linear and nonlinear mixed effects models. r package version 3.1-137 (2018)

31. H. Wickham, ggplot2: Elegant Graphics for Data Analysis (Springer-Verlag New York, 2016). URL https://ggplot2.tidyverse.org

32. L.P. Teunissen, A. Grabowski, R. Kram, Journal of Experimental Biology 210(24), 4418 (2007)
33. C.T. Farley, T.A. McMahon, Journal of Applied Physi- ${ }_{486}$ ology 73(6), 2709 (1992)

34. C.R. Taylor, N.C. Heglund, T.A. McMAHON, T.R. ${ }_{488}$ Looney, Journal of Experimental Biology 86(1), 9 (1980)

35. C.J. Arellano, R. Kram. Partitioning the metabolic cost of human running: a task-by-task approach (2014)

36. T.D. Royer, P.E. Martin, Medicine and science in sports 492 and exercise 37(4), 649 (2005)

37. R.W. Selles, J.B. Bussmann, A.K. Van Soest, H.J. Stam, 494 Disability and Rehabilitation 26(12), 694 (2004) 